\title{
Therapeutic Effects of SB 365 Natural Aticancer Drug for Various Cancers with Advanced Stages in 7 Years
}

\author{
Jong-Hwa Lee ${ }^{1}$, Joo-Yeon Gie ${ }^{2}$, Yoo-Jin Cho ${ }^{3}$, Chul-Soo Kim ${ }^{1,3}$, Myung-Seup Chae ${ }^{4}$, \\ Euishin Edmund Kim ${ }^{5,6, *}$, Wang-Jae Lee ${ }^{7}$, U-Hyun Park ${ }^{8,9}$, Thomas Lau ${ }^{10}$, Josef Sotckenreiter ${ }^{9}$, \\ Stefan Woehrer ${ }^{11}$, Karl Postbauer ${ }^{12}$ \\ ${ }^{1}$ Sahmook Cancer Ceter, Sahmyook Seoul Hospital, Seoul, Korea \\ ${ }^{2}$ Department of Radiology, Sahmyook Seoul Hospital, Seoul, Korea \\ ${ }^{3}$ Department of Internal Medicine, Sahmyook Seoul Hospital, Seoul, Korea \\ ${ }^{4}$ Department of Family Medicine, Sahmyook Seoul Hospital, Seoul, Korea \\ ${ }^{5}$ Graduate School of Convergence Science and Technology, Seoul National University, Seoul, Korea \\ ${ }^{6}$ Department of Radiological Sciences, University of California at Irvine, California, USA \\ ${ }^{7}$ College of Medicine, Seoul National University, Seoul, Korea \\ ${ }^{8}$ Department of Medical Oncology, Kyunghee University Hospital, Seoul, Korea \\ ${ }^{9}$ Department of Internal Medicine, East-West Intergrative Medicine Hospital, Wien, Austria \\ ${ }^{10}$ Department of Medicine, Paracelsus Intergrative Hospital, Lustmuhle, Switzerland \\ ${ }^{11}$ Cancer Center, Neustadt State Hospital, Wien, Austria \\ ${ }^{12}$ Department of Medical Oncology, University Hanover Hospital, Hanover, Deutschland
}

\section{Email address:}

edmundek@hs.uci.edu (E. E. Kim)

${ }^{*}$ Corresponding author

\section{To cite this article:}

Jong-Hwa Lee, Joo-Yeon Gie, Yoo-Jin Cho, Chul-Soo Kim, Myung-Seup Chae, Euishin Edmund Kim, Wang-Jae Lee, U-Hyun Park, Thomas Lau, Josef Sotckenreiter, Stefan Woehrer, Karl Postbauer. Therapeutic Effects of SB 365 Natural Aticancer Drug for Various Cancers with Advanced Stages in 7 Years. Journal of Cancer Treatment and Research. Vol. 9, No. 3, 2021, pp. 50-55. doi: 10.11648/j.jctr.20210903.12

Received: May 28, 2021; Accepted: June 17, 2021; Published: July 19, 2021

\begin{abstract}
SB is a root extract of plant Pusatilla koreana, used traditionally as an useful antiinflammatory and antitumor agent in South Korea for long times. From March 2013 to December 2019, for 7 years there were 421 consecutive advanced and recurrent various kinds of cancer patients for intensified SB 365 anticancer drug treatment. Two important components, Pulsatilla saponin D is the most effective saponin with cancer cell apoptosis, deoxypodophyllotoxin inhibits new blood vessel formation, and anti-inflammatory effects with immune potentiative activities were confirmed. Cancer studied firstly in pancreas, followed by stomach, large intestine, lung, bile duct, liver, breast, kidney, uterus, oral cavity, esophagus and prostate. Almost of cancers were advanced and recurred status. Intravenous administration was done with intratumoral injection, intraperitoneal or intrathoracic administrations were done with change position, and inhalation therapy was also done. Side effects were clinically insignificant, most commonly fever, chills, nextly injection site pain, erythema, itching, nausea and vomitings were developed. $73.6 \%$ of total patients received 1-2 cycles of chemotherapies only, 3 cycles received patient survived for 6 months, 6 cycles survived for 12 months, and 9 cycles or more survived 30 months of survival durations. Pain control with improved appetite and immunity were noted as SB 365 treatments were going on. It is strongly requested to regain the permission of Korean FDA and prompt supports of the national medical insurance, that makes remarkable survival duration and for lesser economic burdons for advanced, recurrent cancer patients and save the funds of national medical insurance. And also highly recommended to make SB treatment available in foreign cancer patients for economic advantages and national image promotions.
\end{abstract}

Keywords: SB 365, Saponin D, Deoxypodophyllotoxin, Natural Anticancer Drug 


\section{Introduction}

SB is a root extract of Pulsatilla koreana that has have been used traditionally as an useful anti-inflammatory and antitumor agent in South Korea for more than 30 years [1, 2]. From March 2013 to December 2019, there were 421 consecutive advanced and recurrent various cancer patients diagnosed radiologically and histologically, admitted to Sahmyook Seoul Hospital for intensified SB 365 anticancer drug treatment.

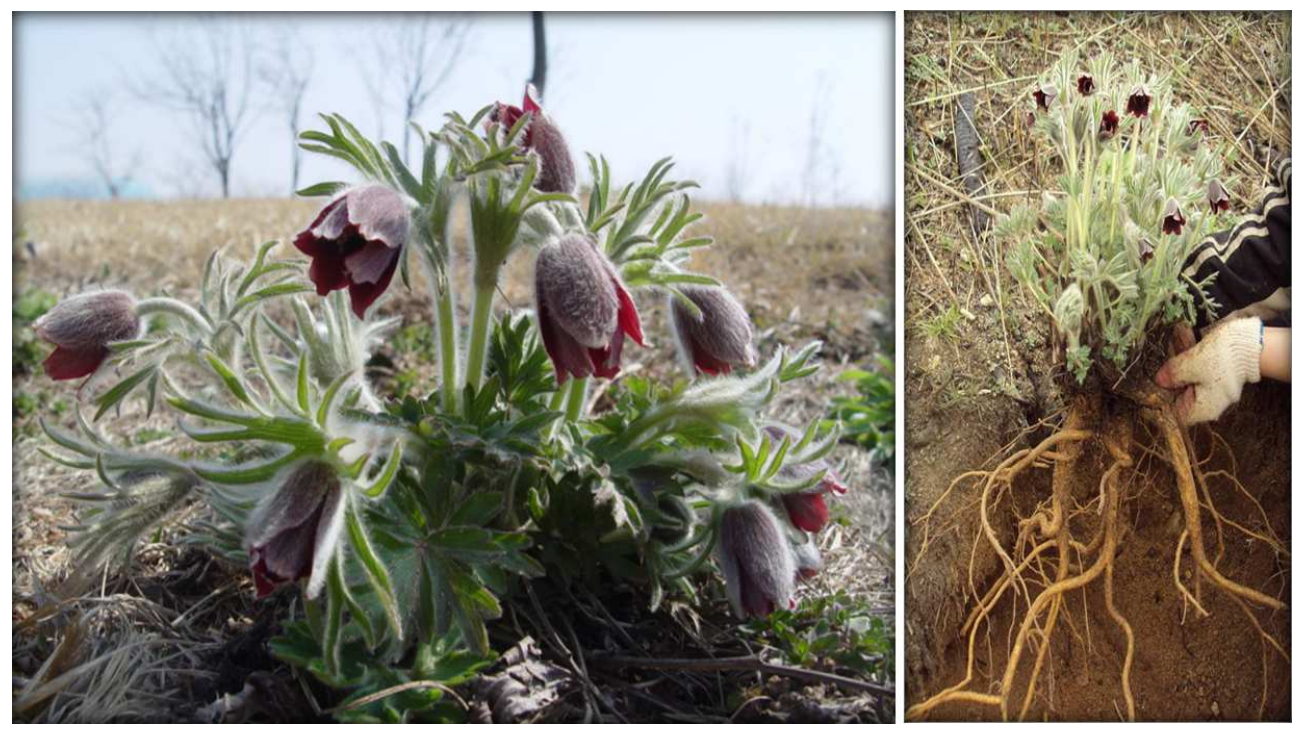

Figure 1. Images of Pulsatilla Korena and their roots.

\subsection{There Are Two Important Components from the Roots of Pulsatilla Koreana}

The most effective saponin for cancer cell apoptosis was identified. In animal model, with the dose of $6.4 \mathrm{mg} / \mathrm{kg} / \mathrm{day}$, $82 \%$ of cancer cells were inhibited, better than the adriamycin that had $60 \%$ of inhibition of pancreatic cancer cells with its dose of $0.5 \mathrm{mg} / \mathrm{kg} /$ day [3].
No cytotoxicity at concentration of $5 \mathrm{ug} / \mathrm{ml}$ and should be $0.8 \mathrm{mg} / \mathrm{vial}$ and more [4].

Molecular structure is $\mathrm{C}_{22} \mathrm{H}_{22} \mathrm{O}_{7}$ as shown below. Showed an inhibition of new blood vessel formation at $20 \mathrm{mg} / \mathrm{kg} /$ day $[3,4]$, and also suppress c-Met signaling in various cancer cell proliferation and angiogenesis $[5,6]$. Anti-inflammatory effects [7] with immune potentiative activity were also confirmed $[8,9]$.

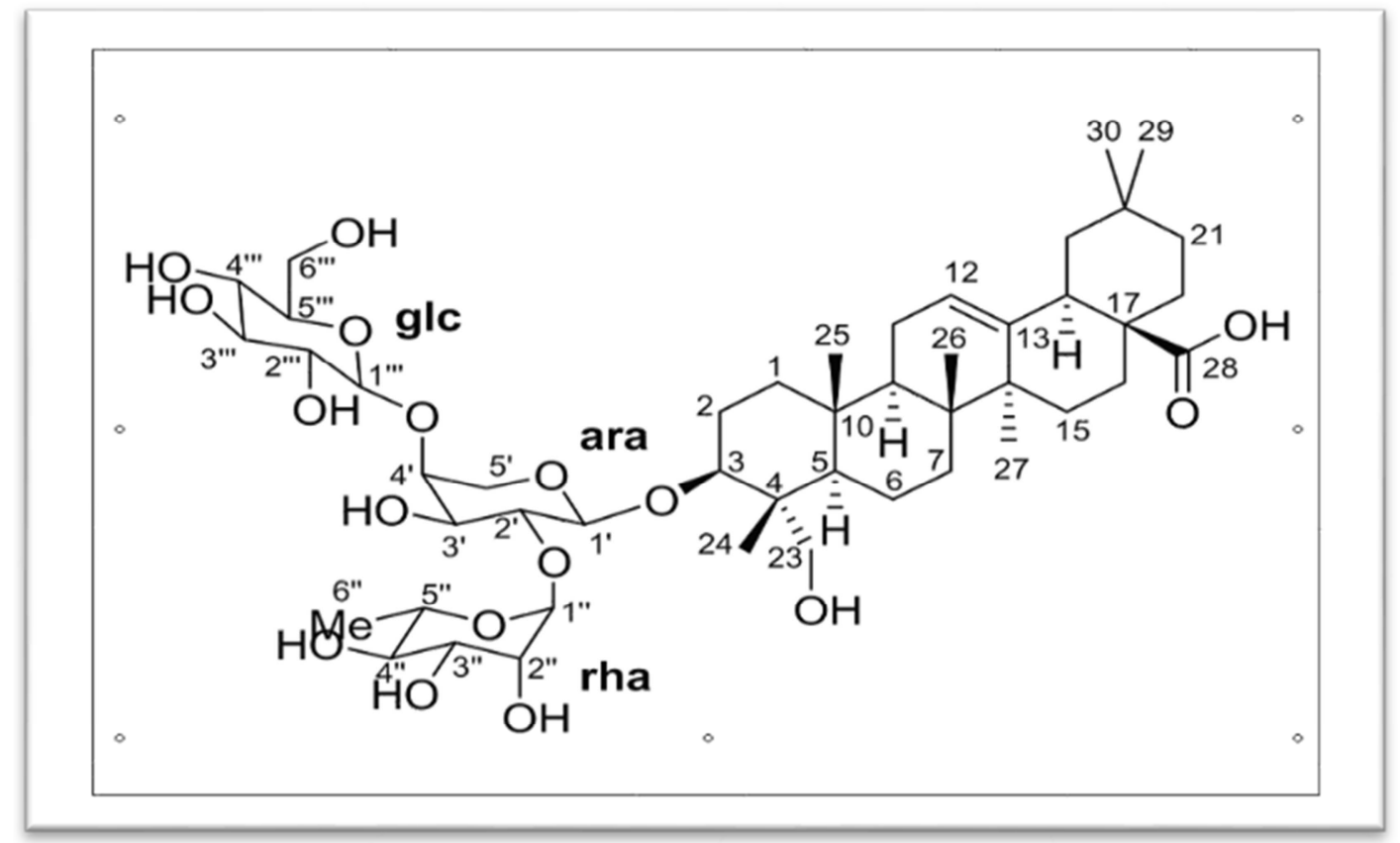

Figure 2. Pulsatilla saponin D (SB 365) Molecular structure is $\mathrm{C}_{30} \mathrm{H}_{46} \mathrm{O}_{4}$ as shown. 


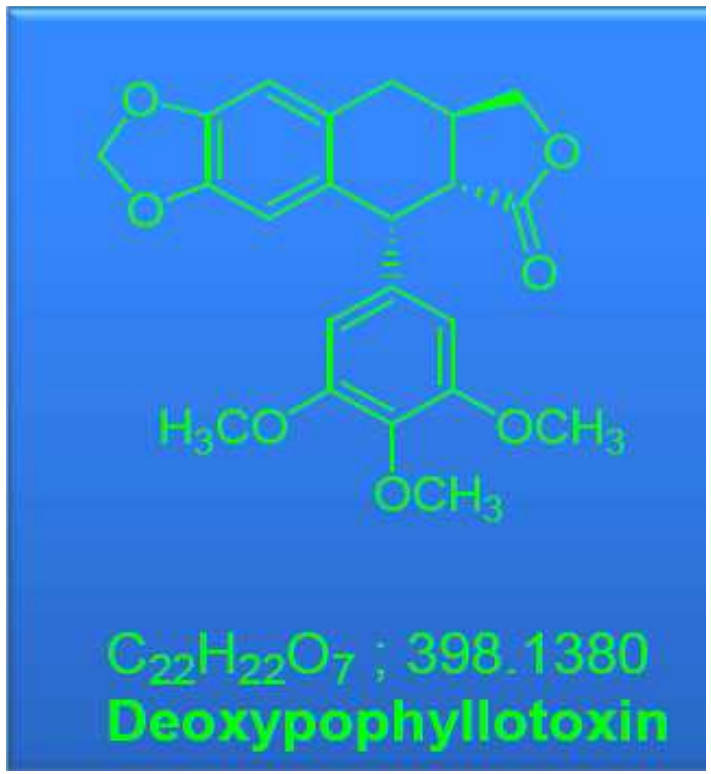

Figure 3. Molecular structure of Deoxypodophyllotoxin.

Table 1. Showing characteristics of enrolled patients (From March 2013 to December 2019).

\begin{tabular}{ll}
\hline Variable & Case \\
\hline No, of Cases & 421 \\
Age range (median) & $12-87(59)$ \\
Gender & \\
Male & $216(51.3 \%)(60)$ \\
Female & $205(48.7 \%)(59)$ \\
& $402(95.8 \%)$ recurred: $14(3.0 \%)$ \\
Cancer stage 4 & Stage $3: 2(0.5 \%)$ Stage $3: 2(0.5 \%)$ \\
& Stage $0: 1(0.2 \%)$ \\
\hline
\end{tabular}

Among 421 SB treatment cancer patients, 67.2\% (283 patients) received standard anticancer therapy. Only surgical resection were done in 37 patients $(8.8 \%)$, and the surgery with chemotherapy and radio therapy were done in 20 patients (4.8\%), Surgery with chemotherapy were done in 95 patients $(22.6 \%)$ and the surgery with radiotherapy were done in 16 patients $(3.5 \%)$. Chemotherapy alone were received in 97 patients $(23.0 \%)$, and the chemotherapy with radiotherapy were done in 10 patients $(2.4 \%)$. Only radiotherapy were done in 6 patients $(1.3 \%)$.

Table 2. Showing cancer patients treated with SB 365.

\begin{tabular}{llll}
\hline Organ involved & No of Patients & Male & Female \\
\hline Pancreas & 119 & 50 & 69 \\
Biliary & 26 & 14 & 12 \\
Liver & 25 & 20 & 5 \\
Stomach & 50 & 30 & 20 \\
Colon & 52 & 25 & 27 \\
Lung & 36 & 27 & 9 \\
Breast & 23 & 0 & 23 \\
Ovary & 12 & 0 & 12 \\
Uterus & 10 & 0 & 10 \\
Urinary & 12 & 9 & 3 \\
Prostate & 5 & 5 & 0 \\
Oral & 8 & 8 & 0 \\
Esopjagus & 5 & 3 & 2 \\
Duodenum & 2 & 2 & 0 \\
\hline
\end{tabular}

Table 3. Showing various cancers treated with $S B 365$.

\begin{tabular}{llll}
\hline Organ involved & $\begin{array}{l}\text { No of cancer } \\
\text { patients }\end{array}$ & Male & Female \\
\hline Lymphoma & 3 & 0 & 3 \\
Melanoma & 3 & 3 & 0 \\
Kaposl's & 2 & 1 & 1 \\
Skin & 2 & 2 & 0 \\
Scalp & 2 & 1 & 1 \\
Neck & 2 & 1 & 1 \\
Retroperitoneum & 2 & 1 & 1 \\
Brain & 5 & 2 & 3 \\
Neural & 3 & 1 & 2 \\
Muscle & 2 & 1 & 1 \\
Thyroid & 2 & 2 & 0 \\
Liposarcoma & 1 & 1 & 0 \\
Thorax & 1 & 1 & 0 \\
Leukemia & 1 & 1 & 0 \\
Osteosarcoma & 1 & 1 & 0 \\
\hline
\end{tabular}

\subsection{Cancer Studied Organs and Clinical Stages}

Pancreatic cancer was first and were preclinically and clinically studied $[10,11]$, followed by cancer of stomach [6], large intestine [12, 13], bile duct, liver [14, 15], breast, ovary, kidney, uterus, oral cavity, thyroid [16], brain [17] (Table 3, 4).

Almost all cancers were advanced 4th stages and recurred cancers, that comprised of $98.8 \%$ of all studied patients (Table 1 ).

\subsection{Methods and Administration Doses of SB 365}

Intravenous administration of the drug, melting 1 vial of SB 365 by $5 \mathrm{ml}$ of $0.9 \%$ normal saline, 6 th grade dose of 21.87 $\mathrm{ml}$ per 1 square meter body surface area everyday was made. Intratumoral injections of the drug was performed under the guidance of ultrasound or CT and, each SB vial was melted with $2.5 \mathrm{ml}$ saline solution, several times according to the size of tumor in each cycle. Intraperitoneal or intrathoracic administrations of the drug, 2-4 vials melted by $10-20 \mathrm{ml}$ saline was made with positional changes every 10 minutes for 1 hour for even distribution. Inhalation therapy was given with SB 1 vial with $2 \mathrm{ml}$ of saline, 1-2 times everyday. Each cycle consisted of 4 to 7 days followed by 1 week of rest time, and next cycle was repeated.

\subsection{Types of SB 365 Administration}

Intravenously: 383 cases including 250 cases of only intravenously.

Intratumoral: 123 cases including 23 cases of only intratumoral.

Intravenously and Intratumoral: 98 cases.

Intravenously and intraperitoneal: 24 cases.

Intraperitoneally only: 2 cases.

Intravenously and intrathoracic: 9 cases.

Intrathoracic only: 4 cases.

Intratumoral with intraperitoneal and intrathoracic: 1 case. Intravenously and inhalation: 16 cases.

Inhalation only: 3 cases.

Intravenously and intratumoral with inhalation: 1 case. Intravenously and intrathoracic with inhalation: 1 case. 


\subsection{Toxicities 1 of $S B 365$}

Side effects were various with no clinical significance. By laboratory tests after every SB injection. They were mostly similar to ones in our previous study in SB treatment in patients with advanced pancreatic cancers [11]. Most of them were disappeared at the end of the each cycles, and prevented by proper preparations. Side effects more than grade 2 are shown in Table 4. The most common SB treatment related side effects were fever about $38^{\circ} \mathrm{C}$ and chillness for 1 hour, disappeared between 1-2 hour later. Injection site pain and erythema were next frequent side effects, and resolved quickly. Direct intratumoral injection also made injection site pain for 4 hours, but it was resolved 1 day later.

\subsection{Toxicities 2 of $S B 365$}

After SB injection, systemic itching sensation with local erythema was developed in some patients, but antihistamines effectively controlled them and also could prevent it after SB injection. After 1 hour of SB injecton, some patients complained nausea frequently but vomitings were not frequent, occasionally diarrhea was developed in some patients. Intraabdominal injection made an abdominal pain in some patients controlled by antispasmodic administration. Around 2 hours later of SB injection, occasionally hypotensive symptoms or, hypertensive state occurred, but those were spontaneously resolved. In inhalation therapy patients, some developed hemoptysis, but it was controlled in stable state.

Table 4. Showing Toxicity Profile of SB injection.

\begin{tabular}{lllll}
\hline Symptom and/or sign & NO. & WHO Toxities Grade 2 & Grade 3 & Grade 4 \\
\hline Fever, chill & 95 & 7 & 1 & 0 \\
Injection site pain, erythema & 69 & 12 & 2 & 0 \\
Skin rash, itching & 18 & 16 & 2 & 0 \\
Nausea, vomiting & 11 & 2 & 2 & 0 \\
Abdominal pain & 3 & 0 & 0 & 0 \\
Dyspnea & 2 & 2 & 0 & 0 \\
Headache & 1 & 1 & 0 & 0 \\
Diarrhea & 1 & 1 & 0 & 0 \\
Hemoptysis & 1 & 1 & 0 & 0 \\
Hypotension & 1 & 1 & 0 & 0 \\
Hypertension & 1 & 1 & 0 & 0 \\
\hline
\end{tabular}

\section{Results}

\subsection{Treatment of 421 Cancer Patients with SB 365}

From March 2013 to December 2019, 28 kinds of cancers in 421 cancer patients were tested using 12 various administration methods. 223 patients $(53.0 \%)$ were received only 1 cycle, 75 patients $(17.8 \%)$ received 2 cycles, 45 patients $(10.7 \%)$ received 3 cycles, 26 patients $(5.9 \%)$ received 4 cycles, 8 patients $(1.9 \%)$ received 5 cycles of their treatments. More than 6 cycles were received in 44 patients $(10.7 \%) .6$ cycles in 8 patients, 7 cycles in 6 patients, 8 cycles in 10 patients, 9 cycles in 1 patient, 10 cycles in 2 patient, 11 cycles in 3 patients, 12 cycles in 1 patient and 14 cycles in 12 patient.

\subsection{SB 365 Treatment in Advanced Recurred Pancreatic Cancers}

Among total 119 pancreatic patients, 66 patients (55.9\%) received only 1 cycle of SB therapy with median survival duration for 2 months, 2 cycles in 19 patients (16.1\%) with same 2 months survival. However 3 cycles in 14 patients $(11.9 \%)$ were performed, and their survival time was elongated to 6 months. 4 cycles in 8 patients $(6.8 \%)$ demonstrated 8 months survival. More than 6 cycles in 12 patients $(10.1 \%)$ noted with 12 months of more elongated survival duration.
Table 5. Showing Survival Durations in Pancreatic Cancer Patients According to the Treatment Cycles.

\begin{tabular}{llll}
\hline Therapy cycles & NO. & (\%) & Surival time (month) \\
\hline 1 Cycle & 66 & 55.5 & 2 \\
2 Cycle & 19 & 16.0 & 2 \\
3 Cycle & 14 & 11.8 & 6 \\
4 Cycle & 8 & 6.7 & 6 \\
5 Cycle & 0 & 0 & 0 \\
More than 6 cycles & 12 & 10.1 & 12 \\
\hline
\end{tabular}

\subsection{Analysis of Survival Duration in Total SB Treated Patients}

Treated more than 3 cycle, 45 patients were died in the hospitals and also 63 patients were confirmed death by national medical insurance. Total 108 patients were died and 16 patients still living at this time, 124 patients were analysed to estimate median survival durations. SB treated by 3 cycles in 45 patients showed 6 months survival duration, for 4 cycles in 26 patients with same 6 months and by 5 cycles in 8 patients with same 6 months also. More than 6 cycles treated in 44 patients noted more elongated survival duration of 12 months. More than 9 cycles treated in 19 patients and more than 12 cycles in 13 patients showed both 30 months of significantly elongated survival durations. 16 patients are still living for 15 to 80 months with median survival of 24 months (Table 6). 
Table 6. Showing Survival Durations in Total Patients According to the Treatment Cycles.

\begin{tabular}{llll}
\hline Therapy cycles & NO. & $\mathbf{( \% )}$ & Surival time (month) \\
\hline 1 Cycle & 223 & 53.0 & 2 (pancreatic Ca) \\
2 Cycle & 75 & 17.8 & 3 (pancreatic Ca) \\
3 Cycle & 45 & 10.7 & 6 \\
4 Cycle & 26 & 5.9 & 6 \\
5 Cycle & 8 & 1.9 & 6 \\
More than 6 cycles & 44 & 10.7 & 12 \\
More than 9 cycles & 19 & 4.5 & 30 \\
More than 12 cycles & 13 & 3.1 & 30 \\
\hline
\end{tabular}

\section{Discussion}

SB treatments in patients with advanced and recurrent various cancers durling 7 years were analysed.

a) Side effects were mild clinically insignificant, and disappeared at the end of their treatment cycles. Preventive preparations were effective mostly.

b) Pain control with effective appetite and immunity improvement [18] were noted with good quality of life after SB therapy.

c) After limited permission by Korean FDA on 11 April 1998, effective anticancerous components were intensified, free marketing by 2008 Quick Permission Law, 3 Hospital cared the about 1,500 terminal cancer patients since year of 2013 with $80 \%$ of cancer control effects without significant side effects [19]. Our hospitals could make analysis of SB 365 therapy data durling 7 years.

d) From 2017 November European country hospital have joined SB anticancer therapy for their terminal cancer patients.

e) Despite of advanced and recurrent cancers, 3 cycles with SB 365 drug conserved 6 months or more survival duration, 6 cycles had made 12 months or more survival, 9 cycles or more of therapy made, 30 months of elongated survival time with much good quality of life.

Since SB 365 drug is not available to others there is no comparison with similar works by others. Many cancer patients wanted to start anticancer chemotherapy with strong hopes of their disease eradication, but more than half of them were abandoned and dropped after only 1 cycle of SB 365 treatment, mostly due to economic burdens without supports of national medical insurance. It is strongly requested to regain the permission of the Korean FDA and prompt supports of the national medical insurance, that makes remarkable survival with good quality of lives by utilizing the SB drugs.

\section{Conclusion}

We conclude that SB 365, newly developed Korean natural anticancer and anti-inflammatory drug is safe, cost effective with cheap production and it can also save the funds of national medical insurance.

It is highly recommended to make SB treatment available in foreign countries for cancer patients. It will make economic advantages and will contribute to the national image promotions.

\section{References}

[1] Kang S S. Saponins from the roots of Pulsatilla koreana. Arch. Pharm Res 1989; 12 (1): 42-47.

[2] Kim S Y and Kim S B. Antitumor effect of Pulsatilla koreana in vitro. J Korean Cancer Asso 1994; 26 (6): 959-963.

[3] Kim Y, Bang S C, Lee J H and Ahn B Z. Pulsatilla saponin D: The antitumor principle from Pulsatilla koreana. Arch Pharm Res 2004; 27 (9) 95-918.

[4] Bang S C, Lee J H, Song G Y, Kim D H, Yoon M Y and Ahn B Z. Antitumor activity of Pulsatilla koreana saponins and their structureactivity relationship. Chem Pharm Bull 2005; 53: 1451-1454.

[5] Kim Y, Kim S B, You Y J and Ahn B Z. Deoxypodophylloxin; The cytotoxic and antiangiogenic component from Pulsatilla korena. Plant Medica 2002; 68: 271-274.

[6] Hong S W, Jung K H, Lee H S, Son M K, Yan H H, Kang N S et al. SB 365, Pulsatilla saponin D, targets C-Met and exerts antiangiogenic and antitumor activities. Carcinogenesis 2013; 34 (9): 2156-2169.

[7] Lee S H, Lee E and Ko Y T. Anti-inflammatory effects of a methanol extract from Pulsatilla korena in lipopolysaccharide-exposed rats. BMB Rep 2012; 45 (6): 371-376.

[8] Hwang J S and Kim S H. Proliferation assay of SB injection on T 3. Kim Y, Bang S C, Lee J H and Ahn B Z. Pulsatilla saponin D: The antitumor principle from Pulsatilla koreana. Arch Pharm Res 2004; 27: 915-9184. Bang and B lymphocyte in SD rat splenocytes. Biotoxtech 2001: 667-699.

[9] Lee J S, Kim D H, Lee C M, Ha T K, Noh K T, Park J W et al. Deoxypodophyllotoxin induces a Th 1 response and enhances the antitumor efficacy of a dendritic cell-based vaccine. Immune Netw 2011; 11 (1): 79-94.

[10] Son M K, Jung K H, Lee H S, Lee H S, Kim S J, Yan H H et al. SB 365, Pulsatilla saponin D suppresses proliferation and induces apoptosis of pancreatic cancer cells. Oncology Reports 2013; 30: 801-808.

[11] Moon K S, Ji J Y, Cho Y J, Lee J H, Choi M S and Kim E E. Therapeutic effects of SB natural anticancer drug in 50 patients with stage IV pancreatic cancer. J Cancer Tre and Res 2015; 3 (3): $42-46$.

[12] N M K, Jung K H, Hong S W, Lee H S, Zheng H M, Choi M J et al. SB 365, Pulsatilla saponin D suppress the proliferation of human colon cancer cells and induces apoptosis by modulating the AKT/mTOR signaling pathway. Food Chemistry 2013; 136: 26-33.

[13] Jang W J, Park B D, Jeong G S, Hong S S and Jeong C H. SB 365, Pulsatilla saponin D, suppresses the growth gefinitinib-resistant NSCLC cells with Met amplication. Oncology Report 2014; 32: 2612-2618.

[14] Hong S W, Jung K H, Lee H S, Choi M J, Zheng H M, Son M K et al. Apoptotic and antiangiogenic effects of Pulsatilla koreana extract on hepatocellular carcinoma. Int J Oncology 2012; 40: 452-460. 
[15] Hong S W, Jung K H, Lee H S, Choi M J, Son M K, Zheng H M et al. SB 365 inhibits angiogenesis and induces apoptosis of hepatocellular carcinoma through modulation of P13K/Akt mTOR signaling pathway. Cancer Science 2012; 103: 1929-1937.

[16] Park B H, Jung K H, Son M K, Seo J H, Lee H S, Lee J H et al. Antitumor activity of Pulsatilla koreana extract in anaplastic thyroid cancer via apoptosis and anti-angiogenesis. Molecular Med Rep 2013; 7: 26-3017.
[17] Hong J M, Kim J H, Kim H M, Lee W J and Hwang Y I. SB 365, Pulsatilla saponin D induces caspase-independent cell death and augments the anticancer effect of temozolomide in glioblastma multiform cells. Molecules 2019; 24 (18), 3230 .

[18] Lee J H, Cho Y J, Chae M S, Lee W J, Park U H and Kim E E. Initial effects of the SB natural anticancer drug on the number of NK cells, CD4+ and CD 8+ T lymphocytes in human peripheral blood. J Cancer Tre and Res 2018; 6 (2): 25-30. 